\title{
EVOLUTION OF IDENTITY SIGNALS: FREQUENCY-DEPENDENT BENEFITS OF DISTINCTIVE PHENOTYPES USED FOR INDIVIDUAL RECOGNITION
}

\author{
Michael J. Sheehan ${ }^{1,2}$ and Elizabeth A. Tibbetts ${ }^{1,3}$ \\ ${ }^{1}$ Ecology and Evolutionary Biology, University of Michigan, 830 N. University Avenue, Ann Arbor, Michigan 48109 \\ 2E-mail: mic@umich.edu \\ 3E-mail: tibbetts@umich.edu
}

Received April 15, 2009

Accepted August 11, 2009

\begin{abstract}
Identifying broad-scale evolutionary processes that maintain phenotypic polymorphisms has been a major goal of modern evolutionary biology. There are numerous mechanisms, such as negative frequency-dependent selection, that may maintain polymorphisms, although it is unknown which mechanisms are prominent in nature. Traits used for individual recognition are strikingly variable and have evolved independently in numerous lineages, providing an excellent model to investigate which factors maintain ecologically relevant phenotypic polymorphisms. Theoretical models suggest that individuals may benefit by advertising their identities with distinctive, recognizable phenotypes. Here, we test the benefits of advertising one's identity with a distinctive phenotype. We manipulated the appearance of Polistes fuscatus paper wasp groups so that three individuals had the same appearance and one individual had a unique, easily recognizable appearance. We found that individuals with distinctive appearances received less aggression than individuals with nondistinctive appearances. Therefore, individuals benefit by advertising their identity with a unique phenotype. Our results provide a potential mechanism through which negative frequency-dependent selection may maintain the polymorphic identity signals in $P$. fuscatus. Given that recognition is important for many social interactions, selection for distinctive identity signals may be an underappreciated and widespread mechanism underlying the evolution of phenotypic polymorphisms in social taxa.
\end{abstract}

KEY WORDS: Colony-level benefit, color polymorphism, dominance hierarchy, evolutionary stable strategy, individual recognition, paper wasp.

Phenotypic polymorphisms occur in a wide range of taxa from flowers (Gigord et al. 2001) to fish (Olendorf et al. 2006), although the adaptive value of many polymorphisms is often unclear. Given that selection and genetic drift typically reduce the amount of variation in a population, explaining these striking phenotypic polymorphisms has been a challenge for evolutionary biologists.

A number of explanations for the evolution of phenotypic polymorphisms have been proposed including local adaptation, mutation-selection balance, and negative frequency-dependent selection (Mitchell-Olds et al. 2007). In local adaptation, connected populations each adapt to separate ecological conditions so that individuals within a local population are relatively monomorphic (Hoekstra et al. 2006). Under the local adaptation hypothesis, phenotypic polymorphism within a population results from migration between populations with opposing selection regimes. Migrants with rare phenotypes generally have lower fitness so the polymorphism is a consequence of gene flow, not adaptation 
per se (Yeaman and Jarvis 2006; Mitchell-Olds et al. 2007). Polymorphisms may also arise via the accumulation of deleterious variants affecting phenotypes that have yet to be purged by selection. Although mutation-selection balance has been extensively studied from a theoretical perspective (Zhang and Hill 2005), few empirical studies have directly tested how much phenotypic variation arises from deleterious mutations. Unlike the local adaptation and mutation-selection balance hypotheses, the negative frequency-dependent selection hypothesis posits that rare phenotypes are favored by selection such that polymorphism within populations is adaptive. Although frequency-dependent selection has the potential to be a powerful evolutionary mechanism for the promotion and maintenance of polymorphisms, relatively few studies have documented frequency-dependent selection in natural populations. Documented examples have typically been narrow in scope (Fitzpatrick et al. 2007) or governed by unknown mechanisms (Nosil 2006; Olendorf et al. 2006). To date, there are few examples of widespread ecological and evolutionary processes that may favor polymorphisms.

One promising system for research into the evolution of phenotypic variation is individual recognition. The highly variable phenotypes used for individual recognition are among the most striking examples of phenotypic polymorphism in nature (Fig. 1A). Individual recognition occurs when individuals are able to discriminate among multiple social partners based on unique phenotypic characters (Tibbetts and Dale 2007; Tibbetts et al. 2008). Individual recognition has evolved independently in a wide range of taxa, making it an excellent model for investigating the evolution of phenotypic polymorphism. Efficient navigation of complex social environments depends on individual recognition in a number of diverse species, including Polistes paper wasps (Tibbetts 2002; Sheehan and Tibbetts 2008), Pachycondyla ants (D'Ettorre and Heinze 2005), lobsters (Karavanich and Atema 1998), crayfish (Seebacher and Wilson 2007) as well as many vertebrates (Jaeger 1981; Cheney and Seyfarth 1999; Jouventin et al. 1999; Hurst et al. 2001; Paz-y-Mino et al. 2004; Grosenick et al. 2007). Species use individual recognition to discriminate among social partners in a number of different contexts such as parental care (Jouventin et al. 1999), the recognition of territorial neighbors (Jaeger 1981), and linear dominance hierarchies (Tibbetts 2002).

To date most of the research on individual recognition has focused on the presence or absence of recognition behavior in a given species, whereas relatively little research has focused on the individual being recognized. It is unknown whether individuals are selected to signal their identity with distinctive phenotypes (i.e., via an identity signal) or if observers cue into otherwise neutral phenotypic variation to recognize conspecifics (Tibbetts and Dale 2007). If being memorably different is advantageous, rare phenotypes are predicted to spread via negatively frequency- dependent selection such that individuals who look, sound, or smell unique will be favored (Dale et al. 2001). Even relatively minor benefits associated with distinctiveness can lead to the evolution of identity signals as long as the phenotypes used for recognition are not costly to produce or maintain (Dale et al. 2001).

Here, we experimentally test whether there are benefit associated with the distinctive, recognizable phenotypes used for individual recognition. Specifically, we test whether distinctiveness is beneficial within a linear dominance hierarchy. Within species with dominance hierarchies, individuals with unique, recognizable phenotypes are predicted to benefit by receiving less aggression than indistinguishable individuals (Barnard and Burk 1979; Dale et al. 2001). Both dominants and subordinates are predicted to benefit from distinctive phenotypes. When animals contest a resource (such as food or a position in a dominance hierarchy) both the winner and loser benefit by settling the contest without costly escalation (Maynard Smith and Harper 2003). Although some species possess signals, such as badges of status, that allow contestants to assess relative agonistic ability, such signals are often poor predictors of fighting ability. Individual recognition, however, allows individuals to accurately assess social partners based on the outcomes of prior interactions. Typically, the first encounter between two competing individuals is quite aggressive, as individuals fight to establish their relative dominance ranks. When individuals can recognize each other, aggression typically declines in subsequent interactions because relative dominance ranks have already been established (Dreier et al. 2007; Sheehan and Tibbetts 2008). However, in species lacking individual recognition, aggression is not predicted to decline over subsequent encounters because the relative ranks of social partners are not clear unless individuals engage in new aggressive contests (Barnard and Burk 1979).

We experimentally tested the benefits of distinctive, easily recognizable phenotypes in the paper wasp, Polistes fuscatus, which uses variable facial patterns for individual recognition (Fig 1A., Tibbetts 2002; Sheehan and Tibbetts 2008). In this species, multiple queens often found nests together. The queens cooperate to rear offspring, but they also compete to form a linear dominance hierarchy (West Eberhard 1969; Reeve 1991). Individual recognition is thought to play an important role in mediating aggressive dominance interactions among wasp queens and aiding colony stability (Tibbetts 2002). We set up groups of four unrelated wasp queens: three wasps with a similar, common appearance and one with a distinctive, rare appearance (Fig 1B) and then compared the interactions of individuals with common and rare appearances.

We make a number of specific predictions about how distinctiveness will influence social interactions. First, distinctive individuals are expected to be more easily identifiable than nondistinctive individuals. Therefore, individuals with a unique 
A

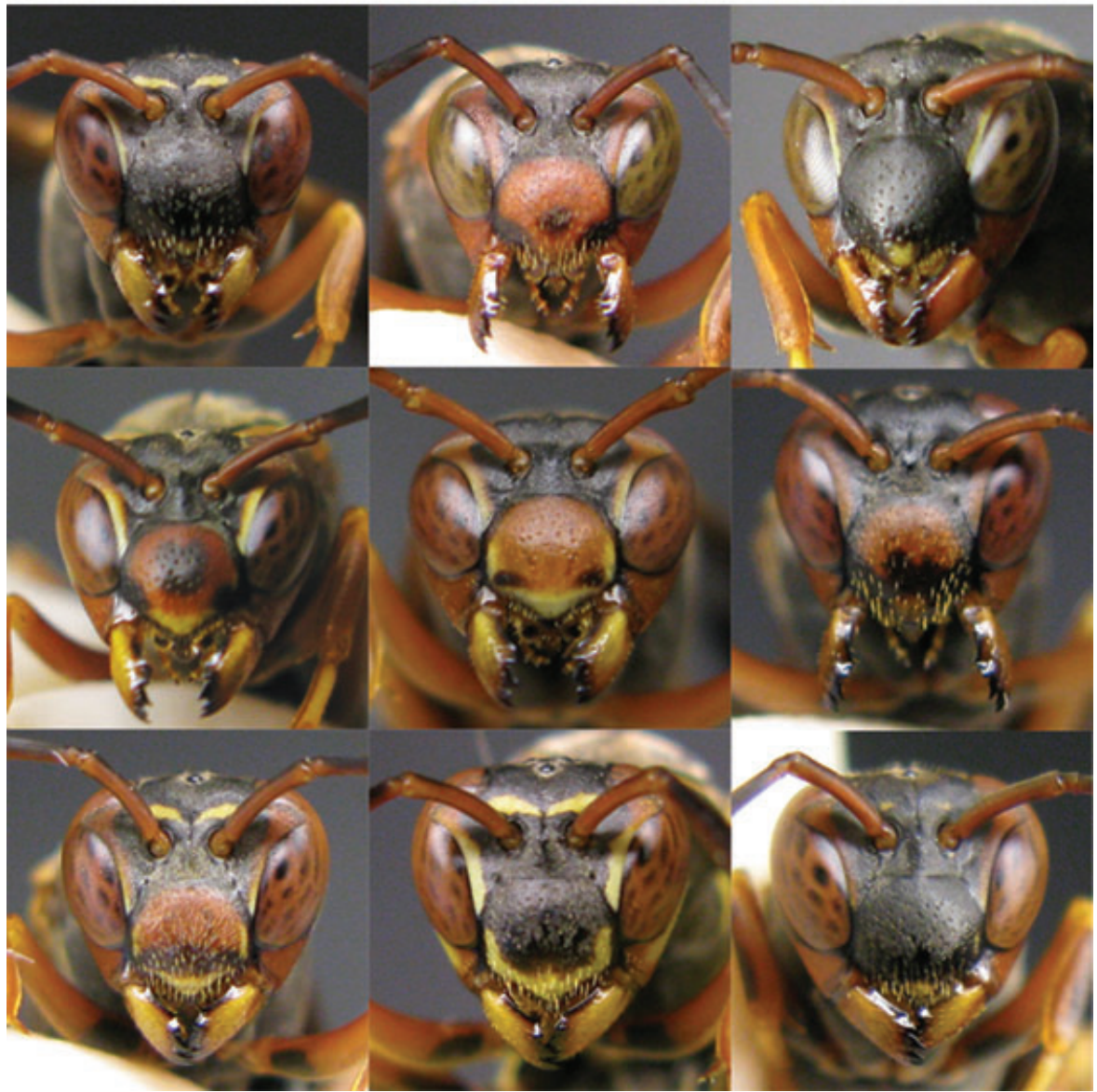

B

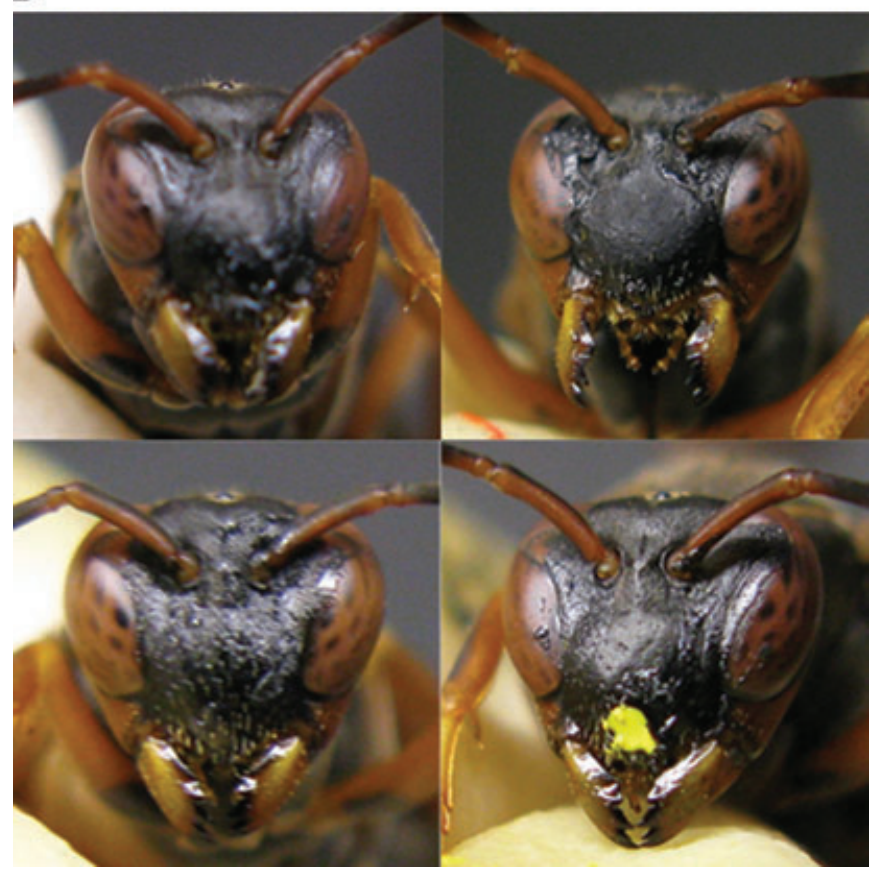

Figure 1. (A) Polistes fuscatus use highly variable facial markings for individual recognition. (B) Examples of wasps with experimentally altered facial patterns. The wasp in the lower right hand corner has a yellow dot on its clypeus. Ten trials contained three wasps with black clypeus tips: one wasp with a yellow clypeus tip whereas eight trials contained one wasp with a black clypeus tip: three wasps with yellow clypeus tips. 
phenotype are predicted to receive less aggression than individuals with a common phenotype. Second, individuals are predicted to have difficulty determining the relative ranks of individuals with common appearances, so the amount of aggression an individual initiates is expected to depend on the number of distinctive versus common individuals they interact with. As a result, wasps that interact with three nondistinctive individuals will initiate more aggression than wasps that interact with two nondistinctive and one distinctive individual.

\section{Methods}

In early spring 2007, behavioral interactions were observed among 18 groups of queens collected from distant locations separated by at least $1 \mathrm{~km}$ around Ann Arbor, Michigan. Each group contained four foundresses that had never previously encountered each other. This mimics natural foundress behavior in early spring. After Polistes foundresses emerge from diapause, they interact with many individuals before settling down to start a nest, either alone or with other foundresses that may or may not be related (Reeve 1991; Queller et al. 2000). Within each group, we painted three wasps to have similar appearances and one wasp to have a unique appearance. The experimental manipulation of markings created a situation in which only one individual (i.e., the unique wasp) was recognizable, allowing us to test the benefits of having a phenotype that allows for individual recognition. Unlike the quality signals found in Polistes dominulus (Tibbetts and Dale 2004; Tibbetts and Lindsay 2008; Zanette and Field in press) and Polistes satan (Tannure-Nascimento et al. 2008), the variable patterns in $P$. fuscatus are not correlated with condition (Tibbetts and Curtis 2007) and do not convey information about their bearer's agonistic abilities. Nevertheless, to ensure that behavioral responses resulted from distinctiveness rather than a particular color pattern, the same color patterns were used for distinctive and nondistinctive wasps in different trials. In half the trials, the distinctive wasp had one yellow facial spot whereas in the other half of the trials; the common wasps had one yellow facial spot (Fig. 1B). These color patterns mimic naturally occurring morphs of P. fuscatus (Tibbetts 2002). The initial experiment that found individual recognition in $P$. fuscatus did so by altering the color of small portions of the face, such as the tip of the clypeus (Tibbetts 2002), indicating that the wasps are able to distinguish between the treatments. Research on other social insects, such as honey bees, has shown that Hymenopteran visual systems are well developed and can easily distinguish among complex patterns (Stach et al. 2004). We chose the distinctive wasp randomly, so the neutral expectation is that the distinctive wasp will be just as likely to receive the most aggression from the other group members, as it is to receive the least aggression from the other group members.
For each trial, we chose four wasps with similar weight (within $0.015 \mathrm{~g}$ ) and general body coloration. To allow identification by the experimenters, each wasp was marked with two small red dots in slightly different locations on the top of the thorax. Given the position and coloring of the red dots the markings are unlikely to increase the distinctiveness of wasps, as wasps do not see red (Briscoe and Chittka 2001). Any additional variation provided by the markings would only dampen the effect of the experimental treatment. After allowing the paint to dry, wasps were placed in an $8 \mathrm{~cm} \times 8 \mathrm{~cm} \times 2 \mathrm{~cm}$ sized container and filmed for $2 \mathrm{~h}$ (see Supplementary information for an example video). The paint treatments remained on all of the wasps' faces throughout the trials. Each wasp participated in only one trial.

MJS watched the tapes without knowledge of the specific experimental treatment. The actor and the recipient were noted for each aggressive act. All aggressive acts including darts, lunges, bites, grapples, and mounts were recorded (West Eberhard 1969). Aggressive behaviors in social wasps are stereotyped, so researchers score these same suites of aggressive behaviors across studies (Reeve and Nonacs 1992; Tibbetts 2002; Strassmann et al. 2004; Weiner et al. 2009). To ensure that there was no subconscious observer bias; MJS trained an individual with no knowledge of the experimental treatment or expected outcomes to score behavioral data. The naive observer watched 12 5-min samples of video from various trials. There was nearly perfect agreement between the initial received aggression ranks found by MJS those found by the naïve observer (Cohen's Kappa with quadratic weighting, $k=0.87$ ), demonstrating that the results are robust across observers (Landis and Koch 1977).

We analyzed the relative distribution of aggression using two complimentary methods. First we asked whether distinctive wasps engaged in a different number of aggressive interactions than nondistinctive wasps. Because the levels of aggression differed among trials (mean $=254.44 \pm 50.01$, range $=120-1049$ aggressive acts), we standardized aggression scores within each trial. To calculate the score, we subtracted the mean number of aggressive acts received or initiated in a trial from the number received or initiated by the wasp of interest and divided this by the standard deviation in aggression scores of that trial. The standard aggression scores for distinctive wasps could then be compared to the population average (set to 0 ) with one sample $t$-tests (Sokal and Rohlf 1995). Second, we considered the relative distribution of aggression. Within each trial, wasps were ranked 1 (most aggression received) to 4 (least aggression received) to analyze the distribution of aggression across all the trials. Because the distinctive wasp was chosen randomly the null expectation is that they should receive the most aggression in one-fourth of the trials, second most in one-fourth of the trials, and so on. We conducted a Monte Carlo simulation of the multinomial sampling distribution for 50,000 iterations. This procedure samples the probability that 


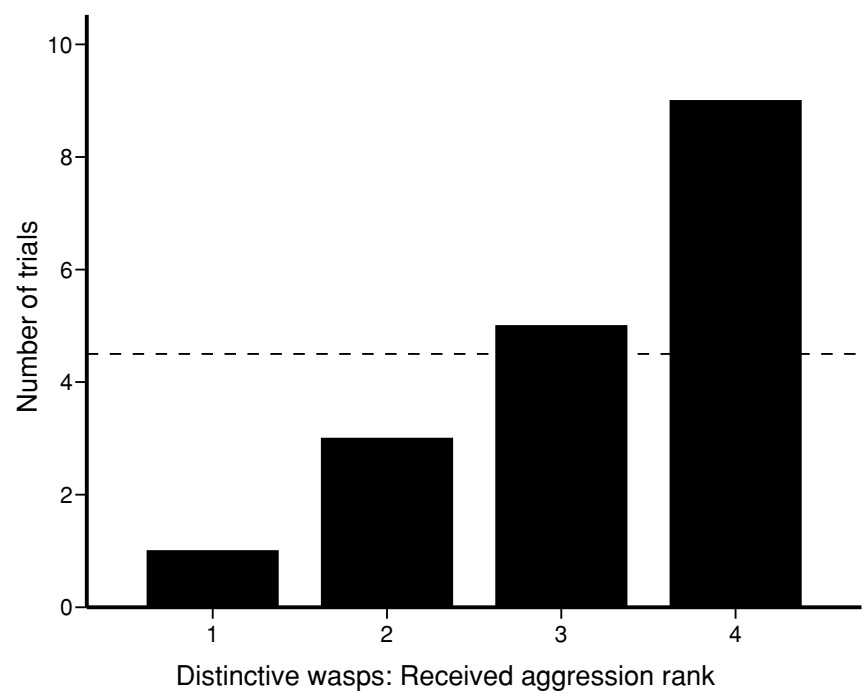

Figure 2. Wasps with distinctive phenotypes (i.e., the rare morph) benefit from advertising their identity. Distinctive wasps received the least aggression in a disproportionate number of trials (multinomial sampling distribution, $N=18, P=0.038$ ). In each trial, wasps were ranked based on the total number of aggressive acts they received from 1 (most) to 4 (least). The dotted line illustrates the null expectation if aggression had been distributed randomly. Only the ranks of distinctive wasps are shown.

a given distribution would occur compared to expected values and approximates a chi-square goodness-of-fit test with a sufficiently large number of iterations. Means are shown \pm SEM and all tests described are two-tailed.

\section{Results}

Rare, easily recognizable phenotypes provided a benefit during social interactions, as the distinctive wasps received less aggression than the population average (mean aggressive acts received $=$ $0.43 \mathrm{SD}$ less than the population $+0.15 \mathrm{SE}$, one sample $t$-test, $\left.t_{17}=-2.95, P=0.0089\right)$. The color treatment did not affect the amount of aggression that distinctive wasps received (two sample $t$-test, $t_{16.0}=1.05, P=0.31$ ). Therefore, aggression was influenced by whether a wasp was distinctive rather than the individual's particular color pattern. Because distinctive wasps were chosen randomly among four individuals, the null expectation is that they should receive the most aggression in one-fourth of the trials and the least in one-fourth. The distribution of aggression, however, was skewed. Distinctive wasps received the least aggression in a disproportionate number of trials (Fig 2. multinomial sampling distribution, $N=18, P=0.038$ ), providing additional evidence that individuals with distinctive phenotypes benefit by receiving less aggression.

The amount of aggression initiated by an individual depended on the number of distinctive versus common individuals they in-

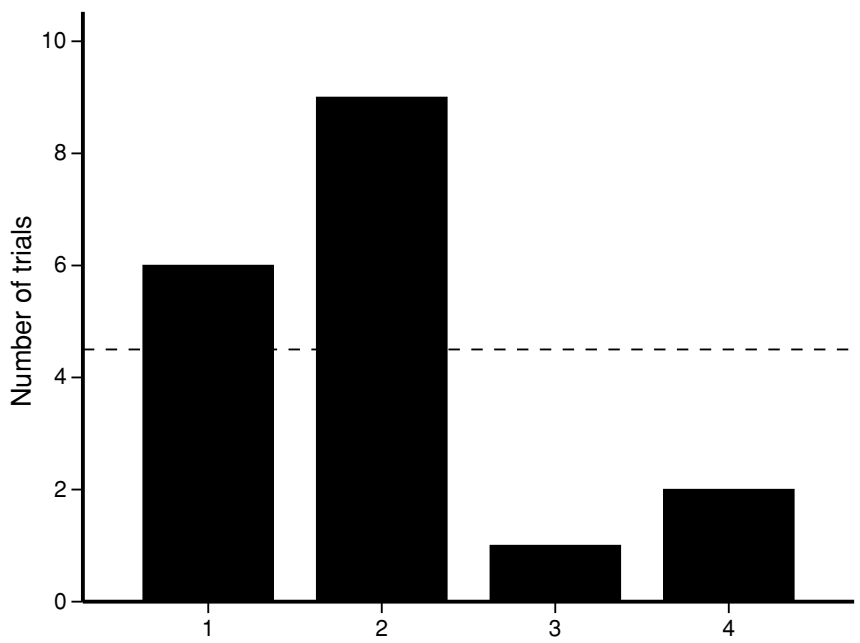

Encounter 3 common wasps: Initiated aggression rank

Figure 3. Wasps with distinctive phenotypes encountered three wasps with the same appearance, whereas common wasps encountered two wasps with the same appearance. Wasps that encountered three individuals with the same appearance were the most aggressive in a disproportionate number of trials (multinomial sampling distribution, $N=18, P=0.033$ ). The dotted line shows the null expectation if aggression had been initiated randomly with respect to the number of common versus distinctive individuals each wasp interacted with. Only the ranks of distinctive wasps are shown.

teracted with. Distinctive wasps interacted with three unidentifiable individuals during each trial whereas common wasps interacted with two unidentifiable individuals and one distinctive individual. As a result, distinctive wasps were more aggressive than the population average (mean aggressive acts initiated $=$ $0.38 \mathrm{SD}$ more than the population $\pm 0.17 \mathrm{SE}$, one sample $t$ test, $\left.t_{17}=2.18, P=0.044\right)$. There was no relationship between the amount of aggression a wasp initiated and the wasp's color, i.e., yellow spot or all black (two sample $t$-test, $t_{14.7}=0.42, P=$ 0.68 ). When considering the distribution of aggression, distinctive wasps initiated the most aggression in a disproportionate number of trials (Fig 3. multinomial sampling distribution, $N=18, P=$ $0.033)$.

\section{Discussion}

Overall, our results provide experimental evidence that individuals benefit when they advertise their identities with rare, distinctive phenotypes. Wasps with distinctive, easily recognizable appearances received less aggression than wasps with common indistinguishable appearances. Given that receiving aggression is costly (West Eberhard 1969; Reeve 1991; Maynard-Smith and Harper 2003) these results indicate that distinctive phenotypes are beneficial. In addition, our results indicate that identity signaling likely provides a colony-level benefit in wasps. Wasps that 
interacted with indistinguishable social partners were more aggressive than individuals that interacted with distinctive individuals. Thus, identity signaling plays an important role in mediating the distribution of aggression in animal societies by allowing aggression to be targeted appropriately. Our experimental results demonstrate that there are benefits associated with having a rare phenotype as well as interacting with individuals that have rare phenotypes. Taken together, these results provide a mechanism through which selection may have driven the evolution of variable visual features in P. fuscatus: negatively frequency-dependent selection.

\section{EVIDENCE FOR IDENTITY SIGNALS}

Prior research on individual recognition has primarily focused on recognition behavior, with little research testing how selection acts on the phenotypes of individuals being recognized (Tibbetts and Dale 2007). Models for the evolution of identity signals critically predict that recognizable individuals will benefit by advertising their identities (Dale et al. 2001), and our results support the predictions of these models.

Traits that evolved to signal individual identity are expected to have a number of properties that distinguish them from traits that evolved to convey other types of information (Dale 2006). Specifically, identity signals are predicted to (1) be highly variable with multimodal frequency distributions, (2) show low to no correlations between traits, (3) be uncorrelated with fitness, (4) expressed independently of condition, and (5) show a high degree of genetic determination (Dale 2006). Any phenotype that fits this specific set of criteria is a plausible candidate for an identity signal.

Previous work indicates that $P$. fuscatus facial patterns fit the predictions of identity signaling models. (1) The facial patterns are highly variable (e.g., Fig. 1A) with a multimodal frequency distribution and (2) no correlation among traits (Tibbetts 2002). (3) The color variation does not correlate with indicators of fitness. Tibbetts (2002) failed to find any relationship between facial patterns and aspects of quality in nest founding queens such as founding strategy, dominance rank, or weight. (4) The facial patterns are also expressed independent of condition. Experimental manipulation of larval nutrition had no effect on the development of P. fuscatus color patterns (Tibbetts and Curtis 2007). (5) Finally, facial patterns are more similar within a nest than between nests. This similarity occurs across both workers and gynes (future reproductives), although the different castes are reared in different conditions. Therefore, there is likely to be a heritable component to variation in facial patterns (M. J. Sheehan and E. A. Tibbetts, unpubl. data). Of course, other mechanisms, such as developmental stochasticity (Leimar 2005) can give rise to polymorphisms, so additional research will be important to assess the precise heritability of $P$. fuscatus color patterns. Overall, the tight fit between the theoretical predictions for identity signals and the characteristics of $P$. fuscatus facial patterns suggests that paper wasp facial patterns have likely evolved to signal individual identity.

Comparative work in Polistes provides further evidence that variable color patterns have evolved because distinctiveness is beneficial in certain paper wasp species. The kind of variable color patterns required to signal individual identity have evolved multiple times in Polistes species with complex social interactions and linear dominance hierarchies (Tibbetts 2004). Species with simpler social interactions, however, do not have variable visual markings. Instead, they have a uniform, species typical color pattern with low intraspecific variability. Given that the majority of Polistes species do not have variable coloration patterns (Tibbetts 2004), the conspicuous phenotypic polymorphisms used for identity signaling in $P$. fuscatus are unlikely to be the result of neutral processes. Rather, selection for distinctive identity signals likely drives the evolution of elaborated, recognizable phenotypes.

Although our results demonstrate that individuals can benefit by advertising their identities, social interactions will not necessarily lead to the evolution of phenotypic polymorphism and identity signals. For example, the chemical profiles that Pachycondyla queens use for individual recognition (D'Ettorre and Heinze 2005; Dreier et al. 2007) are not more variable than chemical profiles in ant species lacking individual recognition (Dreier and D'Ettorre 2009). Therefore, observers can cue into variation that has not evolved specifically to signal identity. When being recognizable is neutral, variable features used for individual recognition may be lost. In some circumstances, selection may even favor individuals that conceal their identities (Johnstone 1997).

\section{IDENTITY SIGNALING AND NEGATIVE FREQUENCY-DEPENDENT SELECTION}

Most previous examples of negative frequency-dependent selection focus on systems with a limited number of morphs that are at an evolutionarily stable state. When multiple, evolutionarily stable foraging or mating strategies are maintained in a population via negative frequency-dependent selection, selection maintains the relative frequencies of the strategies at equilibrium (Maynard Smith 1982). In contrast to many other examples of negatively frequency-dependent selection, selection for identity signaling favors individuals with a unique appearance rather than a particular strategy. As a result, it is expected to produce a large array of polymorphic phenotypes. Unique traits are favored because they are useful for discriminating among conspecifics and thus are expected to spread in a population. As a trait increases in frequency, it will become less useful for discriminating among individuals at which point it is no longer expected to spread in the population. Unlike evolutionary stable strategies, new variants are expected to invade the population because they provide individuals with distinctive phenotypes. Over time, this dynamic 
is expected to produce populations with numerous polymorphic traits that are uncorrelated (Dale et al. 2001; Dale 2006), as observed in $P$. fuscatus (Tibbetts 2002). Therefore, selection for identity signaling differs from many other examples of negatively frequency-dependent selection because it favors extremely high variation, rather than maintaining a limited number of morphs at equilibrium.

\section{BENEFITS: INDIVIDUAL OR GROUP?}

Polistes fuscatus paper wasps live in complex social groups where the fitness of an individual is influenced by the productivity of their colony, so distinctive phenotypes may provide benefits for both the individual and the group (Korb and Heinze 2004). Our experiment was designed to test Dale et al.'s (2001) model, which posits that individuals with rare, recognizable phenotypes will benefit by receiving less aggression during social interactions; our results are consistent with this prediction. In most taxa, high levels of aggression are costly, as fighting increases the chance of injury (Jaeger 1981). In Polistes, intense fighting can lead to severe injury including the loss of limbs and wings or even death (West-Eberhard 1969; M. J. Sheehan and E. A. Tibbetts, pers. obs.), suggesting that reductions of aggression through identity signaling may increase individual fitness. Further, individuals that receive intense aggression often reduce other activities, including brood care, foraging, and social interactions. Previous research on individual recognition in $P$. fuscatus indicates that distinctiveness may reduce aggression in a range of contexts. Known individuals receive less aggression than unknown individuals on nests (Tibbetts 2002) and in the laboratory (Sheehan and Tibbetts 2008).

The results of this experiment suggest that there may also be colony-level benefits associated with identity signaling. Individuals that interacted with indistinguishable conspecifics initiated more aggression than those that interacted with more distinctive conspecifics. Further, interacting with distinctive conspecifics may increase colony stability, as distinctiveness allowed individuals to target their aggression appropriately. In natural colonies of $P$. dominulus, lower levels of aggression are associated with higher rates of resource sharing (Tibbetts and Reeve 2000). Whether increased cooperation is a cause of consequence of lower aggression levels is unknown, though at least one theoretical model predicts that individual recognition will increase cooperation (Crowley et al. 1996). Therefore, distinctive phenotypes are likely to be favored at both the individual and colony level in P. fuscatus.

\section{Conclusion}

Overall, selection for identity signals is likely to occur across a range of taxa and sensory modalities. Our results demonstrate that easily recognizable individuals with rare, distinctive phenotypes benefit by receiving less aggression from conspecifics than indi- viduals with common, nondistinctive phenotypes. Highly polymorphic features facilitate recognition in a wide range of taxa from paper wasps to swallows (Medvin et al. 1993) to humans (Kanwisher and Yovel 2006). Further, recognition is an essential component of social interactions across a range of behavioral contexts (Sherman et al. 1997; Mateo 2004; Tibbetts and Dale 2007). Therefore, negative frequency-dependent selection favoring identity signaling is likely to be an underappreciated mechanism for the maintenance of phenotypic polymorphisms in many social taxa. Future research on identity signals is likely to uncover many more social situations in which the benefits of being unique have driven the evolution of phenotypic diversity.

\section{ACKNOWLEDGMENTS}

Many thanks to T. Bergman, M. Izzo, T. Hendry, L. Knowles and J. McCormack, and A.J. Zellmer, and two anonymous reviewers for helpful comments on earlier drafts of this manuscript. T. Hendry and A. Mettler provided assistance collecting wasps. S. Klein watched tapes. Funding was provided by the University of Michigan.

\section{LITERATURE CITED}

Barnard, C. J., and T. Burk. 1979. Dominance hierarchies and the evolution of 'individual recognition'. J. Theor. Biol. 81:65-73.

Briscoe, A. D., and L. Chittka. 2001. The evolution of color vision in insects. Annu. Rev. Entomol. 46:471-510.

Cheney, D. L., and R. M. Seyfarth. 1999. Recognition of other individuals' social relationships by female baboons. Anim. Behav. 58:67-75.

Crowley, P. H., L. Provencher, S. Sloane, L. A. Dugatkin, B. Spohn, L. Rogers, and M. Alfieri. 1996. Evolving cooperation: the role of individual recognition. Biosystems 37:49-66.

Dale, J. 2006. Intraspecific variation in coloration. Pp. 36-86 in G. E. Hill, and K. J. McGraw, eds. Bird coloration, volume 2, function and evolution. Harvard Univ. Press, Cambridge, MA.

Dale, J., D. B. Lank, and H. K. Reeve. 2001. Signaling individual identity versus quality: a model and case studies with ruffs, queleas, and house finches. Am. Nat. 158:75-86.

D’Ettorre, P., and J. Heinze. 2005. Individual recognition in ant queens. Curr. Biol. 15:2170-2174.

Dreier, S., and P. D'Ettorre. 2009. Social context predicts recognition systems in ant queens. J. Evol. Biol. 22:644-649.

Dreier, S., J. S. van Zweden, and P. D'Ettorre. 2007. Long-term memory of individual identity in ant queens. Biol. Letts. 3:459-462.

Fitzpatrick, M. J., E. Feder, L. Rowe, and M. B. Sokolowski. 2007. Maintaining a behaviour polymorphism by frequency-dependent selection on a single gene. Nature 447:210-U215.

Gigord, L. D. B., M. R. Macnair, and A. Smithson. 2001. Negative frequencydependent selection maintains a dramatic flower color polymorphism in the rewardless orchid Dactylorhiza sambucina (L.) Soo. Proc. Natl. Acad. Sci. USA 98:6253-6255.

Grosenick, L., T. S. Clement, and R. D. Fernald. 2007. Fish can infer social rank by observation alone. Nature 445:429-432.

Hoekstra, H. E., R. J. Hirschmann, R. A. Bundey, P. A. Insel, and J. P. Crossland. 2006. A single amino acid mutation contributes to adaptive beach mouse color pattern. Science 313:101-104.

Hurst, J. L., C. E. Payne, C. M. Nevison, A. D. Marie, R. E. Humphries, D. H. L. Robertson, A. Cavaggioni, and R. J. Beynon. 2001. Individual recognition in mice mediated by major urinary proteins. Nature 414 :631634. 
Jaeger, R. G. 1981. Dear enemy recognition and the costs of aggression between salamanders. Am. Nat. 117:962-974.

Johnstone, R. A. 1997. Recognition and the evolution of distinctive signatures: when does it pay to reveal identity? Proc. R. Soc. Lond. B 264:15471553.

Jouventin, P., T. Aubin, and T. Lengagne. 1999. Finding a parent in a king penguin colony: the acoustic system of individual recognition. Anim. Behav. 57:1175-1183.

Kanwisher, N., and G. Yovel. 2006. The fusiform face area: a cortical region specialized for the perception of faces. Philos. Trans. R. Soc. B 361:2109-2128.

Karavanich, C., and J. Atema. 1998. Individual recognition and memory in lobster dominance. Anim. Behav. 56:1553-1560.

Korb, J., and J. Heinze. 2004. Multilevel selection and social evolution of insect societies. Naturwissenschaften 91:291-304.

Landis, J. R., and G. G. Koch. 1977. Measurement of observer agreement for categorical data. Biometrics 33:159-174.

Leimar, O. 2005. The evolution of phenotypic polymorphism: randomized strategies versis evolutionary branching. Am. Nat. 165:669-681.

Mateo, J. M. 2004. Recognition systems and biological organization: The perception component of social recognition. Ann. Zool. Fennici 41:729745.

Maynard Smith, J. 1982. Evolution and theory of games. Cambridge Univ. Press, Cambridge, England.

Maynard Smith, J., and D. Harper. 2003. Animal signals. Oxford Univ. press, New York.

Medvin, M. B., P. K. Stoddard, and M. D. Beecher. 1993. Signals for parent offspring recognition: a comparative-analysis of the begging calls of cliff swallows and barn swallows. Anim. Behav. 45:841-850.

Mitchell-Olds, T., J. H. Willis, and D. B. Goldstein. 2007. Which evolutionary processes influence natural genetic variation for phenotypic traits? Nat. Rev. Genet. 8:845-856

Nosil, P. 2006. Frequency-dependent selection: when being different makes you not stand out. Curr. Biol. 16:R806-R808.

Olendorf, R., F. H. Rodd, D. Punzalan, A. E. Houde, C. Hurt, D. N. Reznick, and K. A. Hughes. 2006. Frequency-dependent survival in natural guppy populations. Nature 441:633-636.

Paz-y-Mino, G., A. B. Bond, A. C. Kamil, and R. P. Balda. 2004. Pinyon jays use transitive inference to predict social dominance. Nature 430:778781.

Queller, D. C., F. Zacchi, R. Cervo, S. Turillazzi, M. T. Henshaw, L. A. Santorelli, and J. E. Strassmann. 2000. Unrelated helpers in a social insect. Nature 405:784-787.

Reeve, H. K. 1991. Polistes. Pp. 99-148 in K. G. Ross, and R. W. Matthews, eds. The social biology of wasps. Comstock, Ithaca, NY.

Reeve, H. K., and P. Nonacs. 1992. Social contracts in wasp societies. Nature 359:823-825.

Seebacher, F., and R. S. Wilson. 2007. Individual recognition in crayfish (Cherax dispar): the roles of strength and experience in deciding aggressive encounters. Biol. Letts. 3:471-474.
Sheehan, M. J., and E. A. Tibbetts. 2008. Robust long-term social memories in a paper wasp. Curr. Biol. 18:R851-R852.

Sherman, P. W., H. K. Reeve, and D. W. Pfennig. 1997. Recognition Systems. Pp. 69-96 in J. R. Krebs and N. B. Davies, eds. Behavioural ecology: an evolutionary approach. Blackwell Science Ltd., Oxford.

Sokal, R. R., and F. J. Rohlf. 1995. Biometry. W.H. Freeman and Company, New York.

Stach, S., J. Benard, and M. Giurfa. 2004. Local-feature assembling in visual pattern recognition and generalization in honeybees. Nature 429:758761.

Strassmann, J. E., A. Fortunato, R. Cervo, S. Turillazzi, J. M. Damon, and D. C. Queller. 2004. The cost of queen loss in the social wasp Polistes dominulus (Hymenoptera : Vespidae). J. Kansas Entomol. Soc. 77:343355.

Tannure-Nascimento, I. C., F. S. Nascimento, and R. Zucchi. 2008. The look of royalty: visual and odour signals of reproductive status in a paper wasp. Proc. R. Soc. B 275:2555-2561.

Tibbetts, E. A. 2002. Visual signals of individual identity in the wasp Polistes fuscatus. Proc. R. Soc. B 269:1423-1428.

- 2004. Complex social behaviour can select for variability in visual features: a case study in Polistes wasps. Proc. R. Soc. B 271:1955-1960.

Tibbetts, E. A., and T. R. Curtis. 2007. Rearing conditions influence quality signals but not individual identity signals in Polistes wasps. Behav. Ecol. 18:602-607.

Tibbetts, E. A., and J. Dale. 2004. A socially enforced signal of quality in a paper wasp. Nature 432:218-222. - 2007. Individual recognition: it is good to be different. Trends Ecol. Evol. 22:529-537.

Tibbetts, E. A., and R. Lindsay. 2008. Visual signals of status and rival assessment in Polistes dominulus paper wasps. Biol. Letts. 4:237-239.

Tibbetts, E. A., and H. K. Reeve. 2000. Aggression and resource sharing among foundresses in the social wasp Polistes dominulus: testing transactional theories of conflict. Behav. Ecol. Sociobiol. 48:344-352.

Tibbetts, E. A., M. J. Sheehan, and J. Dale. 2008. A testable definition of individual recognition. Trends Ecol. Evol. 23:356-356.

Weiner, S. A., W. A. Woods, and P. T. Starks. 2009. The energetic costs of stereotyped behavior in the paper wasp, Polistes dominulus. Naturwissenschaften 96:297-302.

West-Eberhard, M. J. 1969. The social biology of polistine wasps. Museum of Zoology, Univ. of Michigan, Ann Arbor, MI.

Yeaman, S., and A. Jarvis. 2006. Regional heterogeneity and gene flow maintain variance in a quantitative trait within populations of lodgepole pine. Proc. R. Soc. B 273:1587-1593.

Zanette, L., and J. Field. 2009. Cues, concessions, and inheritance: dominance hierarchies in the paper wasp Polistes dominulus. Behav. Ecol. 20:773780 .

Zhang, X. S., and W. G. Hill. 2005. Genetic variability under mutation selection balance. Trends Ecol. Evol. 20:468-470.

Associate Editor: G. Arnqvist

\section{Supporting Information}

The following supporting information is available for this article:

Movie S1. Example video of fighting trials.

Supporting Information may be found in the online version of this article.

(This link will take you to the article abstract).

Please note: Wiley-Blackwell is not responsible for the content or functionality of any supporting information supplied by the authors. Any queries (other than missing material) should be directed to the corresponding author for the article. 